\title{
Circuit
}

Musiques contemporaines

\section{La mesure du temps : un entretien inédit avec Stockhausen (1958)}

\section{The measure of time: An unpublished interview with Stockhausen (1958)}

\section{Karlheinz Stockhausen et Maryvonne Kendergi}

Volume 19, numéro 2, 2009

Stockhausen au Québec

URI : https://id.erudit.org/iderudit/037452ar

DOI : https://doi.org/10.7202/037452ar

Aller au sommaire du numéro

Éditeur(s)

Les Presses de l'Université de Montréal

ISSN

1183-1693 (imprimé)

1488-9692 (numérique)

Découvrir la revue

Citer cet article

Stockhausen, K. \& Kendergi, M. (2009). La mesure du temps : un entretien inédit avec Stockhausen (1958). Circuit, 19(2), 63-76.

https://doi.org/10.7202/037452ar
Résumé de l'article

Entrevue accordée en français par Karlheinz Stockhausen à Maryvonne Kendergi et diffusée le 15 décembre 1958 durant l'émission Festivals européens, sur les ondes de la radio de Radio-Canada. Avec l'aimable autorisation de Madame Kendergi et de la Stockhausen Foundation for Music. 


\title{
Documents
}

\section{La mesure du temps: \\ un entretien inédit avec Stockhausen (1958)}

\author{
KARLHEINZ STOCKHAUSEN ET MARYVONNE KENDERGI \\ TRANSCRIPTION : RÉJEAN BEAUCAGE ${ }^{1}$
}

Le texte qui suit est la transcription d'une entrevue qu'a réalisée Maryvonne Kendergi en 1958 pour son émission hebdomadaire Festivals européens ${ }^{2}$, diffusée sur les ondes de Radio-Canada. II s'agit d'un document d'une rare qualité, qui témoigne d'une époque durant laquelle l'évolution technologique et l'imagination des compositeurs ont fait subir à la musique

1. Nous avons adopté pour cette transcription l'utilisation des majuscules pour la graphie des titres des œuvres de Stockhausen, plutôt que l'habituelle mise en italiques, comme le compositeur avait lui-même choisi de le faire.

2. "Le Festival du dimanche devenu les Festivals européens [1958] tiendra l'antenne pendant sept ans, de l'automne 1956 à la fin du printemps 1963, à raison de plus ou moins 39 émissions par année. En tout, je crois pouvoir dénombrer 243 premières auditions d'œuvres ainsi que 127 interviews de 58 compositeurs et de 69 interprètes. Les émissions, dont la matière provenait des festivals d'Europe, étaient toujours une conjugaison d'œuvres classiques et d'œuvres contemporaines. Graduellement, cependant, le public en est venu à demander davantage de musiques nouvelles. Vœu auquel je souscrivais volontiers, m'intéressant moi-même de plus en plus à leur diffusion." Maryvonne Kendergi, citée dans Bail, 2002, p. 240. "institutionnelle" des mutations importantes, qui allaient s'avérer capitales pour son développement.

Maryvonne Kendergi, née le 15 août 1915 à Aïntab en Cilicie, a étudié le piano à l'École normale de Paris et obtenu un diplôme d'enseignement (1933) avant d'entreprendre une carrière de concertiste (1933-1937) et des études avec Alfred Cortot (1938). Après l'obtention d'une licence complète d'enseignement (1940) et d'une licence libre ès lettres, elle devient déjà l'organisatrice d'activités culturelles que connaîtront plus tard les Montréalais; au Collège franco-britannique de la Cité internationale universitaire de Paris (1945), puis à la Fondation des États-Unis (Head of Cultural Section, 1948). Elle rejoint ses parents à Gravelbourg, en Saskatchewan, en 1952 et, en 1956, retournant à Paris en passant par Montréal, elle y reçoit du directeur du réseau français de la Société Radio-Canada l'offre d'animer la série d'émissions Festival du dimanche.

Parallèlement à une importante carrière de communicatrice à la radio et à la télévision, elle 
occupera une place de premier plan dans le développement du milieu de la musique contemporaine à Montréal. Organisatrice, aux côtés de Pierre Mercure, de la Semaine internationale de musique actuelle de Montréal en 1961, elle sera aussi cofondatrice, en 1966, de la Société de musique contemporaine du Québec (SMCQ). Invitée à enseigner à la Faculté de musique de l'Université de Montréal la même année, elle y offrira les premiers cours consacrés à la musique canadienne dans une université canadienne et y développera la série des "Musialogues", des rencontres devant public avec les grands compositeurs et interprètes de l'époque (1969-1983).

La rencontre avec Karlheinz Stockhausen, dont témoignent les pages qui suivent, marque le premier passage du compositeur à Montréal. Le luxe de précautions dont fait preuve l'animatrice dans sa présentation du compositeur démontre assez la nouveauté de ses propositions pour les auditeurs de cette retransmission, ainsi que l'esprit aventurier dont Maryvonne Kendergi ne s'est jamais départie.

\section{Transcription de l'entrevue diffusée le 15 décembre 1958 durant l'émission Festivals européens, sur les ondes de la radio de Radio-Canada.}

Bonsoir chers amis,

En vous accueillant ce soir à notre émission, je voudrais un instant reprendre plus particulièrement ce rôle d'hôtesse que l'on me donnait naguère, car j'ai à vous présenter non point un programme, mais un compositeur, et un compositeur qui n'est pas comme les autres. Non seulement parce que tout créateur authentique est un être unique, mais aussi parce que l'homme qui double le créateur chez Karlheinz Stockhausen est exceptionnel. Cela, vous le percevrez vite dans ses propos, même si ce qu'il vous dira pourra vous paraître ardu parce que très technique. Et même si sa musique semble déroutante ou hermétique.

Je voudrais donc tout d'abord vous prier instamment de ne pas quitter l'écoute en cours d'émission, vous, cher ami, qui n'aimez que Mozart et les classiques, vous, cher autre ami, qui ne tolérez que difficilement le vocabulaire moderne, vous, enfin, qui êtes bien d'accord avec la musique contemporaine, mais qui n'admettez encore qu'avec réserve le langage dodécaphonique ou refusez le titre de musique à tout ce qui n'est pas instrumental. Vous tous, chers auditeurs, qui nous dites votre fidélité dans la confiance, même si l'étrangeté de certaines œuvres de nos programmes vous déconcerte, gardez l'écoute, car c'est bien pour vous spécialement, pour cette émission des Festivals européens, que Karlheinz Stockhausen est venu à Montréal.

Si je me permets de le mentionner ici, c'est qu'un jour viendra, s'il n'est déjà venu, où nous serons sans doute fiers d'avoir été l'instrument de cette première présence du compositeur parmi nous. Et comme il est de mise entre nous à certaines occasions, laissez-moi vous narrer l'enchaînement des faits. En juin dernier, lors du festival de la Société internationale pour la musique contemporaine à Strasbourg, on donnait une œuvre de Stockhausen. OEuvre et compositeur étaient attendus au festival avec l'impatience que vous devinez, aussi n'était-ce pas sans une certaine réticence qu'après l'exécution de son œuvre sous sa direction, j'allais essayer d'aborder le compositeur. Jeune, ainsi entouré, assailli même par tous les noms chevronnés de la critique musicale européenne, comment allait-il recevoir une commentatrice de radio, même venant du Canada, qui sollicitait une interview? Avec une simplicité désarmante, Stockhausen me dit: "Je repars pour Cologne dans deux heures, mais venez là-bas, et nous ferons quelque chose." "Mais je ne peux pas, dis-je. Je dois repartir sans délai pour Montréal. Mais venez donc 
vous-même au Canada, je suis sûre que les jeunes musiciens en seraient enchantés." "On s'intéresse donc à cette musique là-bas?" demande Stockhausen. "Venez et vous verrez", lui dis-je. "Pourquoi pas? On s'écrira", conclut-il. Et c'est ainsi que, en août dernier, je recevais à ma grande surprise un mot de Stockhausen annonçant qu'au terme d'une tournée de conférences dans les universités américaines, il pourrait venir à Montréal si cela nous intéressait. Le directeur des programmes a immédiatement accepté que toute une émission soit consacrée à la musique électronique et aux œuvres de Karlheinz Stockhausen. À son tour, le compositeur a accepté de venir passer 48 heures parmi nous, prêt, disait-il, à faire ce que nous lui demanderions. Si je vous donne ces détails, chers amis, c'est bien pour vous dire, d'une part, que Radio-Cananda n'hésite pas à poser un geste que Karlheinz Stockhausen lui-même a reconnu comme unique, celui de consacrer un programme de cette durée, et sur tout son réseau, à cette seule production, et cela parce que nous savons à quel auditoire nous nous adressons. C'est aussi, d'autre part, pour vous donner une idée de la disponibilité et du caractère direct de cet être exceptionnel, devant qui le mot "génie" s'impose, mot que je prononce avec tout le respect, et l'admiration, que vous partagerez très vite, j'en suis persuadée, dès que vous entendrez avec quelle puissance de pensée, et quelle intensité de réflexion, ce jeune compositeur apporte à notre $20^{\mathrm{e}}$ siècle le choc et la fécondation de son œuvre créatrice. Ce génie, c'est l'être le plus simple au monde. Intransigeant devant la musique et les exigences de sa propre attitude, il sait aussi, ceux qui l'ont approché ces jours-ci peuvent en témoigner, il sait être simplement celui qu'Antoine Goléa ${ }^{3}$ décrit ainsi, je cite: "Jeune terriblement, grand,

3. Antoine Goléa (1906-1980): musicologue français; l'un des membres fondateurs de l'Académie Charles Cros; l'un des participants de l'émission La tribune des critiques de disques sur France Musique; chroniqueur à Télérama, à Diapason; auteur d'une Histoire du ballet (1967). Source: Wikipédia, 2009 . blond, les yeux très bleus, penché, tendu; écoutant la musique avec une attention sans défaillance. " Ils ont pu le voir ainsi, ceux qui se pressaient jeudi dernier dans la salle 404 de l'Université de Montréal, où la Faculté de musique donnait l'hospitalité au groupe Musique de notre temps qui présentait la conférence-concert de Karlheinz Stockhausen. Ceux-là qui étaient venus, bravant un froid draconien et qui furent nombreux à devoir rester debout pendant près de deux heures, ceux-là ont été récompensés en recevant leur part de communication directe, et s'ils sont à l'écoute ce soir, leur profit en sera décuplé. Et pour nous, qui avons eu le privilège de travailler en studio avec le collaborateur permanent du studio de musique électronique de Cologne, quelle leçon! Quelle grâce oserais-je dire, et vous comprendrez ce terme je crois lorsqu'à votre tour vous percevrez, vous recevrez, chers amis, cette présence vivifiante de Karlheinz Stockhausen.

Mais pour cela, et laissez-moi insister en m'excusant de ce long préambule, laissez-moi vous prier instamment de faire réellement le vide et le silence autour de vous, afin de pouvoir recevoir toute la densité et la profondeur de la présence et du message de Stockhausen, dans ses propos et dans sa musique. Cette dernière a été exécutée au cours de trois grands festivals, soit le KONTRA-PUNKTE à Aix-en-Provence, le ZEITMASZE à Strasbourg, et à Donaueschingen les GROUPES pour trois orchestres. La Radiodiffusion et le Südwestfunk de Baden-Baden nous ont envoyé les enregistrements de ces concerts, mais étant donnée l'importance extrême de la fidélité sonore dans cette musique où les moindres secondes de son ou de silence comptent, fidélité nécessairement entravée par les bruits de salle ou de plein air en exécution publique, nous avons utilisé des enregistrements faits en studio par les mêmes interprètes, enregistrements que le compositeur a bien voulu mettre à notre disposition. Pour ce qui est de l'œuvre électronique GESANG DER JÜNGLINGE, il va sans dire qu'elle ne peut exister autrement que sur bande magnétique; là encore, c'est celle du compositeur que 
nous avons eu la chance d'utiliser, si bien que ce programme prend réellement la valeur d'un document précieux qui vous apporte l'œuvre et le message de celui que, il y a deux jours, j'interrogeais de votre part à nos studios de Montréal.

MK: Karlheinz Stockhausen, je ne pensais pas, lorsque je vous ai vu à Strasbourg en juin dernier, à la suite de l'exécution de votre ZEITMASZE, et en vous jetant cette boutade, "Venez nous voir à Montréal", je ne pensais pas que ça deviendrait une réalité, et c'est merveilleux, maintenant, de pouvoir présenter votre œuvre à nos auditeurs avec votre propre présence, pour nous, aujourd'hui. Et je vais vous poser des questions qui vont vous paraître élémentaires, ou boétiennes, mais je sais que vous aurez la gentillesse de vous y prêter.

KS: Ah! bien sûr! Elles sont toujours plus nécessaires, à mon avis, que les questions des spécialistes, parce qu'ils savent... qu'il faut le faire.

MK: Merci. Nous savons que vous avez 30 ans, et à 30 ans, vous avez déjà une réputation qui vous précède, ou qui vous accompagne, mais voudriez-vous me dire, par exemple, quelle a été la part de l'enseignement que vous êtes allé puiser auprès d'Olivier Messiaen à Paris, quelle a été la part de cet enseignement dans votre système, ou dans votre écriture actuelle?

KS: Je peux dire que l'œuvre de Messiaen m'a intéressé profondément depuis sa quatrième étude pour piano ; ça s'appelle Mode de valeurs et d'intensités [1949]. Quand je l'ai entendue pour la première fois en 1951 à Darmstadt, on avait un disque qu'un critique français avait apporté, c'était une vraie révolution pour moi, parce que je sentais quelque chose qui était l'expression de ce qu'il fallait développer dans mon travail. J'ai écouté ce disque au moins dix fois de suite, et puis j'ai décidé: "Quand tu auras fini de travailler au Conservatoire de Cologne, tu iras immédiatement à Paris." J'y suis allé au début de 1952, et c'était juste après que j'aie écrit ma première composition, le KREUZSPIEL. Aujourd'hui, je ne le joue plus, et je ne l'ai pas publié, mais je trouve toujours que c'est la source de mon travail.

MK: On peut mentionner que cela veut dire "Jeux croisés".

KS: [simultanément] "Jeux de croisements", c'est ça. Et la partie de l'œuvre de Messiaen, qui est toujours restée la plus importante, était justement l'époque où il a écrit ses études de piano, quand il a trouvé la première fois l'essence du nouveau langage, c'est-à-dire d'établir ce que l'on appelle l'égalité des droits entre tous les éléments sonores. Parce que, dans cette étude, qui est assez courte, et Messiaen lui-même n'a même pas tiré les conséquences de cette étude-là, il a déterminé les quatre caractéristiques du son au même plan de la composition. Ça, c'était une vraie révolution. Les hauteurs n'étaient plus les choses les plus importantes dans la musique, c'est-à-dire les mélodies ou l'harmonie, mais toutes les caractéristiques des sons, c'est-à-dire les hauteurs, les durées, les attaques et même, après, dans sa Messe de la Pentecôte [1949-1950], les registres de l'orgue. Après avoir entendu ça, j'étais tranquille, c'est-à-dire je trouvais le chemin pour aller plus loin et tirer les conséquences de cette base spirituelle.

MK: Et voudriez-vous alors, maintenant nous dire quelles ont été les œuvres essentielles de votre production depuis cette date-là, ou bien si vous avez à nous faire partager des événements plus importants dans votre existence artistique?

KS: Oui, vous êtes bien gentille de me faire décider moi-même sur l'essentiel de mes œuvres [rires].

MK: Je ne m'aventure pas à le dire moi-même...

KS: Vous savez, j'ai écrit si peu que je peux dire même toutes les œuvres, c'est-à-dire les CONTRE-POINTS [KONTRA-PUNKTE], en 1953, que j'ai publiés comme première partition, pour 10 instruments, et puis des pièces de piano [KLAVIERSTÜCKE I-IV, 1953], et puis les premières études électroniques [STUDIE I, 1953 et STUDIE II, 1954], et puis le deuxième recueil de PIĖCES POUR PIANO, de V à X, et puis le ZEITMASZE [1955-1956] 
et les GROUPES pour trois orchestres [1955-1957], et puis la XI PIĖCE POUR PIANO [1956] et le GESANG DER JÜNGLINGE [1956], I'œuvre électronique avec une voix d'homme.

MK : Et voici que ce soir nous allons avoir la chance, parmi ces œuvres essentielles - et nous reviendrons peut-être tout à l'heure sur ce mot -, parmi ces œuvres, d'en entendre cinq, ce qui est vraiment un privilège, je crois, pour notre connaissance musicale. Quelle œuvre voudriez-vous nous faire entendre en premier?

KS: Moi, je dirais que l'on commence avec la toute première, c'est-à-dire avec les CONTRE-POINTS, l'œuvre qui était la démarche, pas seulement pour mon travail personnel, mais pour ce que l'on appelle en Europe la "musique ponctuelle", où les éléments de la musique obtiennent une importance fondamentale, et où l'on écoute vraiment des "points sonores" qui sont arrangés dans un nouveau sens de structure; peut-être puis-je donner quelques indications..

MK: Qui vont nous aider à écouter l'œuvre.

KS: Exactement... Pour que vous puissiez vous rendre compte que le langage de la musique électronique et celui de la musique instrumentale est le même. Parce que nous ferons jouer aussi tout à l'heure une œuvre électronique, et on pourra bien faire la comparaison. Lorsque nous avons des visiteurs au studio de Cologne, ils dépassent vite, heureusement, leur stupéfaction en face des sonorités inouïes, et ils commencent à demander pourquoi il n'y a pas de mesure en $3 / 4$, point de mélodie, ni répétition ou reprise, et pourquoi il y a un manque total de tout ce que l'on avait l'habitude de rencontrer dans la grammaire musicale valable jusqu'ici. Moi, je dis que le cas est le même avec la musique instrumentale, c'est le même langage. C'est ainsi que la conversation ne porte plus sur la musique électronique; ce ne sont pas les sonorités qui prennent les auditeurs au dépourvu, mais bien la façon dont elle sont "composées", le langage, le nouveau langage, qui a commencé avec le langage ponctuel. Nous avons fait passer des enregistrements d'œuvres d'Anton Webern, en commençant avec celles de l'année 1910, et les auditeurs ne sont pas moins dépaysés lorsque je leur dis que je considère Webern, qui leur était même inconnu, comme le compositeur le plus important de ce siècle. Ensuite, nous faisons passer des œuvres instrumentales composées après 1950, des œuvres des plus importants compositeurs italiens, comme Nono et Berio, quelques fois de l'Américain Cage ou de Varèse, du Français Pierre Boulez, des compositeurs belges Goeyvaerts, qui a joué un certain rôle au début de ce développement, et Pousseur. Et c'est dans la même intention que je voudrais faire entendre les CONTRE-POINTS. Dans cette composition, tout est déjà intégré dans le nouvel ordre musical, que l'on a appelé l'ordre sériel, sauf les timbres instrumentaux, et c'est l'état juste avant le début du travail électronique, parce que là, on trouvait la possibilité d'intégrer aussi les timbres dans la composition. Mais au début on était encore obligé, comme Webern, ou comme Messiaen, nos deux exemples, on était obligé de les accepter en tant que données différentes; il n'était pas possible d'établir un rapport de parenté entre, par exemple, un son de clarinette et un son de piano. La seule chose possible était d'utiliser les timbres donnés par l'orchestre - qui est établi une fois pour toutes depuis les temps classiques -, quelquefois enrichi par de nouveaux instruments, mais le principe fondamental est toujours resté le même, c'est-à-dire d'employer des timbres, avec le spectre harmonique -, de montrer ces timbres dans une suite de contrastes, qui est toujours un principe de forme le plus primitif possible (le contraste). Comme une suite de couleurs: le bleu, le rouge, le jaune. L'idée de faire éclore tous les aspects divers d'une composition, d'un seul germe susceptible de mettre en évidence qu'un son de clarinette et un son de piano peuvent être conçus comme deux exemplaires d'une plus vaste espèce, d'un continuum de timbres les englobant, de façon que toutes les diversités seraient intégrées, on peut vraiment dire: "Quelle utopie!", en face d'un orchestre classi- 
que. Voici maintenant les CONTRE-POINTS, que j'ai écrits en 1953, au début, pour 10 instruments.

\section{Extrait sonore : KONTRA-PUNKTE}

Suggestion d'écoute

KONTRA-PUNKTE ; London Sinfonietta / K

Stockhausen; enregistrement de juillet 1973;

Stockhausen-Verlag $n^{\circ} 4$.

KS: ZEITMASZE est une composition où je trouvais pour la première fois dans mes compositions la notion de "groupe", c'est-à-dire de faire, pour un groupe de sons, les mêmes caractères; par exemple, un groupe qui reste pour un certain temps dans le registre aigu, dans le registre bas, avec une tendance à passer du bas à l'aigu, ou qui a un certain caractère de densité en face d'un autre groupe; comme ça, une masse plus ou moins dense des groupes est caractérisée par un seul caractère sonore, ils ont les mêmes couleurs, ou la même intensité pour un certain temps, etc. Comme ça, le groupe était le premier pas vers une nouvelle composition, et j'appelle cette nouvelle composition "statistique", parce que quelque fois, on n'entend plus les notes toutes seules, on entend toute une masse, et on ne sait plus où l'on est, c'est-à-dire que le caractère de toute la masse des sons est comme une masse de mouches, dans laquelle on ne peut pas compter toutes les mouches! C'est une masse, comme on ne voit pas chacune des feuilles d'un arbre, mais les contours de l'arbre, sans voir les feuilles séparément. Dans ce caractère-là, on trouve la composition de masses, qui n’a jamais eu d'importance dans la musique du passé, et ça donne, après la musique ponctuelle, une nouvelle signification pour les formes musicales. Au moment où l'on en avait perdu les thèmes, les motifs, il faut trouver une nouvelle chose qui va immédiatement dans la perception et qu'on reconnaît, par exemple, une masse qui descend, après un certain temps, on écoute encore une masse, qui est tout à fait renouvelée dans les sons, dans la couleur et dans l'intensité, mais qui descend aussi. Et ça donne une...

MK: ... un va-et-vient...

KS: Une relation structurelle. Comme ça, on peut entendre le ZEITMASZE, où différentes structures, plus ou moins denses, plus ou moins rapides, apparaissent durant les douze minutes.

MK: Juste avant d'écouter votre œuvre, Karlheinz, voudriez-vous me permettre de vous demander de définir ce mot, "Zeitmaße", qui est un terme allemand. "Mesure de temps", mais...

KS: Exactement, ce sont des mesures de temps et j'ai employé - parce que c'est écrit pour des musiciens dans un nouveau sens d'interprétation - cinq mesures de temps. Première mesure: de jouer une phrase ou un groupe le plus vite possible, selon les possibilités de l'interprétation, et c'est là qu'entre en compte la capacité du musicien qui joue son instrument propre. C'est différent pour une voix ou pour une flûte; c'est une pièce pour cinq instruments: flûte, hautbois, cor anglais, clarinette et basson. Alors, quand j'ai indiqué "le plus vite possible" pour le basson, il a un "plus vite possible" qui est très différent du "plus vite possible" de la flûte, et quand j'ai écrit une phrase dans la tessiture grave, le "plus vite possible» est différent de celui dans une tessiture aiguë, etc. Comme ça, j’ai prévu les différents caractères de "plus vite possible". Le deuxième est le "plus lent possible", c'est-à-dire selon la longueur du souffle du musicien qui joue un groupe, il doit le jouer avec le souffle le "plus lent possible", comme ça il trouve un tempo...

MK: ... à sa mesure.

KS: ... qui dépend du musicien qui le joue. Et je trouve au moment où l'on va écrire dans un nouveau sens pour les musiciens, et pas pour des machines, comme on le fait dans la musique électronique, il faut trouver les possibilités qui lui sont propres. La troisième mesure, c'est de commencer le plus vite possible et de ralentir jusqu'à un tempo quatre fois plus lent. Le 
quatrième c'est le contraire, et le cinquième tempo est le tempo métronomique où j'ai vraiment indiqué ce que j'appelle une octave de temps. Partant du tempo métronomique 6o, jusqu'à 120, j’ai écrit douze tempos, tempi, différents; ce sont les cinq mesures de temps.

MK: Nous allons dire, avant d'écouter l'œuvre par le quintette..

KS: C'est mon groupe de la radio de Cologne, que j'ai dirigé moi-même...

MK: Et qui est vraiment celui qui a créé l'œuvre, et qui l'incarne à votre point de vue, n'est-ce pas?

KS: Oui, nous avons fait l'année dernière à peu près quarante exécutions publiques, et c'est là d'ailleurs où nous nous sommes rencontrés!

MK: C'est cela, exactement, au Festival de Strasbourg. Et nous allons entendre donc cette œuvre à son optimum; merci de nous aider à l'écouter.

\section{Extrait sonore : ZEITMASZE}

Suggestion d'écoute

ZEITMASZE; London Sinfonietta / K.

Stockhausen, enregistrement de juillet 1973:

Stockhausen-Verlag $\mathrm{n}^{0} 4$.

MK: Vous avez prononcé, déjà, à deux ou trois reprises, le terme "musique électronique", et c'est un terme qui est à la mode, non seulement parce que ces temps-ci votre passage à Montréal l'a mis sur toutes les lèvres, mais parce qu'on l'entend de plus en plus fréquemment, et je crois que nous sommes nombreux à ne pas savoir ce que c'est exactement. Puisque nous avons la chance d'avoir le compositeur de musique électronique, voudriez-vous nous expliquer ce que c'est, Karlheinz Stockhausen?

KS: Oui je voudrais bien. Vous savez que la musique électronique existe depuis l'année 1953; elle est née à la radio de Cologne.
MK: Alors, est-ce à dire que vous êtes le premier compositeur de musique électronique?

KS: Oui, la première composition qui a été faite est STUDIE 1 et, tout de suite après STUDIE 2, que j'ai réalisées en 1953 à Cologne. Nous avons commencé les opérations du studio vers ce temps-là, avec M. Herbert Eimert, qui est toujours le directeur du studio.

MK: Alors, pouvons-nous dire que les essais du Club d'essai, du laboratoire de Pierre Schaeffer à la Radiodiffusion française, n'étaient pas de la musique électronique?

KS: Non, j'avais travaillé un an avant à Paris dans le Groupe de musique concrète, et là on ne travaille uniquement qu'avec des sons enregistrés - enregistrés avec des microphones - et après on les transforme par des moyens électriques, électro..., électroacoustiques.

MK: Oui. Et maintenant, vous allez nous dire ce que c'est que la musique électronique, justement par opposition à la musique concrète.

KS: D'accord. On peut se demander comment on en est arrivé à cette musique. Partant de la connaissance des partitions écrites jusqu'alors, quelques musiciens se proposaient de remettre en question encore une fois tout ce qui constitue la musique, comme je l'ai dit tout à l'heure, à commencer par les sons eux-mêmes. On avait fait l'expérience que l'évolution historique des sons et les instruments employés, des instruments classiques jusqu'ici, avaient été liés à une musique qui n'est plus la nôtre. On a bien eu l'idée de dire autre chose, mais on se servait du même vocabulaire. C'est ainsi que l'on est arrivé à la découverte de la contradiction fondamentale qui existe entre les sons instrumentaux donnés, d'une part, et les nouveaux concepts formatifs de la musique de l'autre. Tandis que le matériau sonore et les instruments se trouvaient en accord intime avec la formation musicale elle-même, dans la musique classique, ou, pourrait-on dire, dans la musique harmonique, cette unité a été détruite par la musique de douze sons. C'est 
pour cela qu'elle est en apparence impure, parce que l'on opérait avec un matériau de façon à nier sa nature même. Les rapports harmoniques et mélodiques entre les sons dans une musique dodécaphonique n'avaient plus rien en commun avec les rapports, comme je le dis, "microacoustiques", à l'intérieur des sonorités instrumentales. Qu'est-ce que ça veut dire? Le musicien se demandait en quoi réside la différence entre les diverses sonorités. Un violon, un piano, ou la voyelle "a", un bruit de vent, ou une consonne, etc. En 1951 et 1952, j'ai fait beaucoup d'analyse, justement au Groupe de musique concrète à Paris. Des sons instrumentaux, des complexes sonores et des bruits naturels. On se servait des appareils électroacoustiques pour se rendre compte de ce qui constitue une sonorité donnée. Le musicien parla de "spectre sonore". On a examiné les complexes sonores l'un après l'autre en employant des filtres électriques, comme on analyse la lumière à l'aide d'un prisme, pour comprendre la nature des phénomènes. Et le musicien se trouvait donc devant la nécessité d'élargir son métier - parce que, bien sûr, on n'a pas appris ces choses au conservatoire -, et de devenir acousticien pour connaître plus profondément son matériau, car il voulait le dominer. Et ceci ne pourra être épargné à aucun compositeur à l'avenir, il ne pourra se contenter de prendre les sonorités qu'on lui donne toutes faites, mais au contraire il refusera une dictature du matériau, et ça c'est fondamental je trouve. II refuse la dictature du matériau; il veut plutôt faire pénétrer ses concepts formatifs le plus profondément possible, jusque dans les sons mêmes, afin d'arriver de nouveau à un accord entre les microstructures acoustiques à l'intérieur d'un son même, et des sons employés dans une composition musicale, les macrostructures qu'il a composées avec ces sons-là, les macrostructures musicales. Après avoir reconnu la nature des sonorités comme quelque chose de déjà préformé, sortant des instruments classiques, et de la manière de les jouer, on a conçu l'idée de se passer de l'emploi des sonorités préformées, l'idée, donc, de composer les sons mêmes.
MK: En réalité, vous voulez aller à la source même de la création, vous voulez le droit de créer le son à son origine.

KS: Si on veut, oui. C'est-à-dire que même les sons sont le résultat de la composition. Et cela, en bref, est devenu possible techniquement. Pendant mon séjour au Groupe de musique concrète à Paris, j'avais acquis par des travaux d'analyse une connaissance de la nature physique du son. J'en avais uniquement le point de départ à Paris, comme je l'ai dit, à transformer par des moyens électriques les bruits et les sons naturels. En 1953, nous avons commencé l'installation du studio de musique électronique; "musique" électronique, pas des décors sonores ou quelque chose comme ça: "musique». Et on mettait en marche les recherches de production synthétique des spectres sonores par des moyens purement électriques. Et à côté des premières œuvres que j'ai écrites comme KONTRA-PUNKTE, on s'essayait aux premières compositions électroniques par des études d'un matériau sonore d'origine purement électroacoustique.

On peut se demander: "Comment arrive-t-on, techniquement, à de telles sonorités?" II y a des dizaines d'années déjà que l'on possède des générateurs électroacoustiques qui produisent des sons purs, que l'on appelle des sons sinusoïdaux. Si on pense à un son instrumental, nous avons dit qu'il se définit par un spectre, c'est-à-dire une oscillation fondamentale avec ses vibrations partielles [partiels]. II y a bien des vibrations partielles, et dans quelle distribution, dans quelle intensité respective dans un spectre, dans quel ordre successif d'entrée dans le temps, et de cessation; tout cela permet de distinguer les spectres les uns des autres. Une vibration sinusoïdale serait donc justement ce qu'est un son partiel au sein d'un spectre: une vibration simple, sans résonance par sympathie. Et une telle vibration ressemble à un son de flûte, qui n'a, lui aussi, presque pas de partiels. De pareilles vibrations sinusoïdales étaient les premiers éléments que les musiciens composaient (comme le mot le dit: 
composer, "mettre ensemble"), différents spectres sonores d'après les exigences d'une œuvre définie. Et c'est ainsi qu'une seule sonorité représente déjà le résultat d'un acte compositionnel. Le compositeur luimême détermine tous les différents paramètres, ou caractéristiques si on préfère, pour arriver finalement à un seul son; quelque fois, on travaille toute une journée pour arriver même à un seul son, pour mettre ensemble... pour en faire la synthèse.

MK: Voudriez-vous me permettre de vous arrêter pour préciser que ce son est produit par vous-même, du moins par le générateur, que vous ordonnez, et ce son recueilli du générateur, vous pouvez le reprendre et le re-traiter à votre gré?...

KS: Sur bande magnétique.

MK: ... sur bande magnétique.

KS: C'est ça, il est enregistré et le travaille de montage, etc., commence.

MK: Cette précision nous aide à comprendre ce que vous venez de dire.

KS: Et on me demande souvent: "Mais comment jouet-on de ces instruments?" Mais vous comprenez donc qu'il n'y a point d'instrument joué par un interprète qui interprète une partition. Les sons sont produits comme à la chaîne d'une usine, puis vient le montage des pièces de ruban, et l'élimination de l'interprète dans ce domaine a été parfois regrettée. Doit-on encore porter le deuil pour l'artisan d'hier qui fabriquait des chaussures à la main, alors que nous avons pris l'habitude de porter des chaussures fabriquées en usine? Ce n'est plus une question qui se pose à nous; le fait est là : en musique électronique, l'interprète n'a plus de fonction. Le compositeur lui-même, en collaboration avec les techniciens, réalise en studio l'œuvre en entier. Par conséquent, cette musique ne peut se faire entendre qu'au moyen de haut-parleurs. Peut-être devrions-nous maintenant donner un exemple, et je voudrais bien donner en exemple ma toute dernière composition électronique, et cette composition porte le titre CHANT
DES ADOLESCENTS. Je l'ai composée en 1955 et 1956. Cette composition a permis, pour la première fois dans mon travail, d'établir la liaison continue entre des sons chantés ([amusé] ne riez pas!), et des sons produits par des moyens électriques.

MK: Je vais vous dire pourquoi je ris: c'est parce que vous voulez, dans la musique électronique, éliminer l'interprète, mais vous l'avez vous-même mis là-dedans...

KS: Oui, mais n'oubliez pas, ces quatre années de travail m'ont permis de trouver une nouvelle liaison entre les sons, naturels comme on peut dire, les sons organiques, et les sons synthétiques, ou artificiels. Et je crois que plus on avance, plus on se rend compte que l'on ne doit pas faire de différence morale entre les médiums, c'est-à-dire que quand j'utilise une machine, un magnétophone et un générateur, ce serait mieux ou pareil que d'utiliser un instrument. On a besoin de déterminer les sons dans leur couleur, et au moment où l'on trouve le moyen d'intégrer un son naturel à l'intérieur d'une famille de sons plus vaste, qui sont produits électroniquement, pourquoi refuser un son tel, surtout qu'on en a besoin, par exemple, pour l'intelligibilité du son, c'est-à-dire de créer un mot dans la composition musicale.

MK: Ce que vous venez de dire est très important Karlheinz Stockhausen pour, peut-être, définir votre propre éthique. C'est que, pour vous, la musique est une nécessité, ce n'est pas une mécanique pure, et quand vous y intégrez la voix humaine, c'est quand même que, pour vous, c'est la création qui compte.

KS: Bien sûr.

MK: Et l'humain n'est pas exclu de votre création.

KS: II ne faut jamais oublier, après l'imagination totale d'une œuvre, que l'on a besoin de certains éléments et de certaines méthodes et de certains médiums. Et ça dépend, quelquefois on a besoin d'un instrument, ou d'une voix humaine, ou d'un générateur électrique, mais ce sont des moyens, rien d'autre, qui servent à 
l'homme pour s'exprimer, pour créer une forme musicale.

Alors, dans GESANG DER JÜNGLINGE, il faut dire que les voyelles étaient, pour moi, des spectres harmoniques, et les consonnes pouvaient être assimilées à des bruits. Et toute une gamme de sons complexes, intermédiaires, a été composée d'une manière synthétique, entre les voyelles et les consonnes. Comme ça, j'établis ce que l'on appelle un continuum. Dès lors, les phénomènes chantés apparaissent comme des cas particuliers, comme certains éléments d'origine exceptionnellement organique, chantés par un petit garçon de douze ans (il a chanté les syllabes, et puis j’ai monté les syllabes ensemble avec des sons électroniques d'un ensemble plus vaste de phénomènes sonores finement contrôlés, c'est-à-dire électroniques, et j'ai mis les sons chantés à l'intérieur de cet ensemble plus vaste). Parfois, ces sons chantés prennent une signification compréhensible, et parfois, au contraire, leur fonction demeure purement sonore, et entre ces deux extrêmes, il existe différents degrés d'intelligibilité. J'avais en tête, vraiment, ce rêve de faire, de créer, un mot par exemple, à partir des sons. Et de créer la langue, le langage, non on peut dire la langue, à partir d'une composition purement musicale, avec des sons qui n'ont pas une intelligibilité au sens..

MK: ... du verbe.

KS: Oui, du verbe. Et entre ces deux extrêmes, il existe différents degrés d'intelligibilité; une série de degrés d'intelligibilité. Cependant, lorsque des signes purement musicaux se changent momentanément en paroles, c'est toujours pour chanter... et ça, c'est ma propre vision du monde... c'est pour chanter la loi de Dieu. J'ai pris des mots du troisième Livre de Daniel, ça s'appelle "Le chant des trois jeunes gens dans la fournaise ardente".

MK: Je suis sûre que les auditeurs se posent une question : à ce moment-là, est-ce exprès que vous avez choisi ce texte-là, ou n'importe quel texte aurait-il pu vous servir d'argument?
KS: Non. Pour moi personnellement, c'était la première fois que j'employais la voix dans la musique, c'està-dire des mots compréhensibles, et quand j'emploie un groupe de sons qui devient compréhensible au sens de la langue, c'est seulement pour... justement pour... pour dire que la seule chose qui est importante, dans la langue, pour moi, et compréhensible dans la communication humaine, c'est justement la loi de Dieu. Alors, je crois que nous allons maintenant écouter l'œuvre.

MK: II ne nous reste plus que ça à faire.

\section{Extrait sonore : \\ GESANG DER JÜNGLINGE \\ Suggestion d'écoute \\ GESANG DER JÜNGLINGE: \\ Stockhausen-Verlag $n^{\circ} 3$.}

MK: GESANG DER JÜNGLINGE a été votre œuvre électronique capitale Karlheinz Stockhausen et, depuis cinq ans, vous vous êtes consacré à la musique électronique, dites-vous, mais vous n'écrivez pas que de la musique électronique?

KS: Non. Après cinq ans de travail, même après trois ans et demi, quatre ans, on trouve que la musique qui était écrite avant pour les musiciens doit être renouvelée dans un sens tout à fait nouveau, c'est-à-dire: le musicien, avant 1953, ou même dans le travail de mes amis Boulez ou Nono, encore en ce moment, le musicien est devenu, de plus en plus, un remplaçant pour une machine, parce que l'on a écrit des choses tellement difficiles, des partitions à l'écriture de plus en plus compliquée, que finalement il n'y avait plus la possibilité de faire une interprétation dans le vrai sens du mot, et c'est normal que l'on ait finalement pris aussi, par ce côté-là de l'évolution musicale qui a commencée déjà avec les premiers chiffres métronomiques chez Beethoven et qui est devenue de plus en plus compliquée pour les musiciens, qu'on a pris la machine pour atteindre la précision voulue. Et j'ai trouvé, maintenant que l'homme a trouvé les moyens pour résoudre 
tous les problèmes techniques, mécaniques, pour définir la structure des sons à l'intérieur des sons, la couleur des sons, toute exactitude dans le temps, dans les intensités, tout ça avec des mesures électroniques (on mesure la bande, le ruban, en centimètres pour mesurer le temps; on mesure en décibels pour mesurer l'intensité, non plus en forte ou mezzo forte; on mesure les hauteurs en fréquence, en nombre de fréquences, de vibrations par seconde, etc. ce sont toutes des données techniques). Maintenant, il faut trouver les vraies sources humaines, quand le musicien joue et que l'auditeur est là. Un exemple: la onzième PIĖCE POUR PIANO (KLAVIERSTÜCKXI), qui a fait la démarche de tout un nouveau développement de la musique interprétée. Là, il n'est plus question de tout ce que l'on fait avec la musique électronique; on donne ce que j'appelle le choix contrôlé, ou le hasard contrôlé dans la musique interprétée. C'est-à-dire que le compositeur indique différents champs de choix, qui sont plus ou moins larges, dans une même composition, où le musicien qui joue, spontanément, sur le moment, prend parti à la création d'une œuvre. Et je vais vous donner une description de cette œuvre-là : sur une grande feuille, j’ai écrit 19 groupes de sons, des groupes de différents caractères, et de différentes densités, etc. Après chaque groupe, j’ai indiqué trois choses. Premièrement, un tempo, et j'ai indiqué les tempi comme ça: tempo 1, tempo 2, tempo $3,4,5,6$, et il peut faire l'échelle des tempi comme il veut, mais il faut qu'il ait six tempi différents. Puis il y a une indication pour les intensités, aussi relatives, et une indication pour le mode d'attaque avec des symboles graphiques. Maintenant, j'ai donné les directions pour l'interprétation de sorte que lorsqu'il commence l'interprétation de la pièce, il voit la feuille et le premier groupe qu'il voit, c'est celui qu'il joue, librement en ce qui concerne le tempo, l'intensité et le mode d'attaque, mais après avoir joué ce premier groupe, il lit les indications et il cherche le prochain groupe. II ne sait pas lequel ce sera, chaque groupe est possible, mais moi j'ai indiqué, j'ai contrôlé avant toutes les combi- naisons possibles, et il joue ce deuxième groupe selon les indications qu'il a lues juste avant, à la fin du premier groupe, et c'est comme ça qu'il joue le troisième groupe, puis le quatrième, et il peut revenir assez tôt, ou plus tard, au même groupe; à ce moment-là, j'ai des indications entre parenthèses qui renouvellent le groupe, il y a des sons qui sont ajoutés ou des sons qu'il laisse tomber, etc., et les mains sont transposées dans un autre registre. Et puis, lorsqu'il revient pour la troisième fois à un groupe, il ne joue plus, parce que ce serait la première répétition, et c'est ainsi qu'une réalisation est terminée. De cette façon, on peut avoir mille, et beaucoup plus...

MK: Ah oui, c'est illimité, n'est-ce pas, comme possibilités?

KS: ... d'interprétations, et chaque interprétation est unique et demande à l'homme qui l'interprète d'être vivant, et ça, c'est quelque chose, pour un musicien, qui participe dans un nouveau sens à la création, et souvent on me demande si c'est de l'improvisation, mais non, il n'y a rien qui est improvisé, c'est-à-dire qui est inventé sur une base qui est faite par le compositeur, comme dans le jazz ou la musique baroque. Rien n'est inventé sur le moment, l'interprète réagit seulement, avec son subconscient, comme un être humain...

MK: ... vivant.

KS: ... vivant.

MK: Oui, ce n'est pas un robot; votre interprète est associé à votre œuvre de création.

KS: Et je crois profondément que nous avons maintenant trouvé un nouveau sens à la musique qui est écoutée par des auditeurs et créé à chaque instant, à côté de la musique électronique qui a totalement une autre direction. Alors je crois que nous allons maintenant entendre une version de la onzième PIÈCE POUR PIANO

MK: Voudriez-vous nous dire qui est l'interprète, simplement. 
KS: Ça, c'est David Tudor, le merveilleux pianiste américain.

\section{Extrait sonore : KLAVIERSTÜCK XI}

Suggestion d'écoute

KLAVIERSTÜCK XI (4 versions), par David Tudor:

enregistrements de septembre 1958;

Hat Hut Records (hatART CD 6142).

\section{ou}

- KLAVIERSTÜCK XI (2 versions), par Ellen Corver; enregistrements réalisés entre le 19 avril 1997 et le 23 février 1998 ; Stockhausen-Verlag n ${ }^{\circ} 56$ A-C.

MK: Après cette KLAVIERSTÜCK XI, qui est de 1956, ce sera notre dernière œuvre. The last, but not the least, n'est-ce pas? Je me rappelle qu'à Donaueschingen vous nous aviez placés, l'auditoire, entre trois orchestres.

KS: Oui.

MK: Qu'est-ce que cela signifiait? D'ailleurs, cela va peut-être même nous expliquer le titre de votre œuvre: GRUPPEN für drei Orchester.

KS: Vous savez, depuis le GESANG DER JÜNGLINGE, un développement tout à fait nouveau a commencé dans la création musicale en Europe. Parce que, ce que l'on appelle la cinquième dimension du son, on l'a introduit dans la composition, et ça, c'est la projection spatiale, comme c'est le cas pour LE GESANG DER JÜNGLINGE qui a été écrite pour cinq groupes de haut-parleurs qui sont placés autour du public au moment où l'on donne l'œuvre. Et la composition des GROUPES pour trois orchestres, je l'ai commencée en 1955. Interrompue à plusieurs reprises, elle se poursuivit jusqu'à l'année 1957. Dès le début du travail se fit sentir la nécessité de faire jouer simultanément plusieurs complexes sonores de tempos différents. Afin de rendre cette polyphonie jouable et perceptible, un grand orchestre de 109 musiciens fut divisé en trois petites formations. Chacune de celles-ci a son propre chef d'orchestre et doit être suffisamment séparée des autres dans l'espace d'écoute.
L'importance et la composition des trois orchestres sont sensiblement égales. On compte parmi chacun d'eux des bois, des cuivres, des instruments à sons pincés et d'autres à sons frottés. Chacune de ces quatre familles instrumentales est à nouveau subdivisée en un groupe à hauteurs précises et un groupe à sons indéterminés. Le passage du son au bruit contrôlé est assuré dans chaque famille par divers instruments à percussion, de bois, de métal ou de peau. Utilisés de manière adéquate, des instruments comme le piano, le célesta, le jeu de timbres [glockenspiel] ou les cloches de vache garantissent également une liaison entre le domaine des sons harmoniques et celui du bruit. C'est toujours le même principe, comme vous voyez. La similitude d'effectifs des trois orchestres répondait au désir de faire voyager les ensembles sonores d'un groupe instrumental à l'autre, de distribuer entre ceux-ci des structures de caractères apparentés, etc. Chaque orchestre devait pouvoir en appeler un autre, lui donner la réponse, ou lui faire écho. En ce qui touche la sonorité orchestrale de cette œuvre, il ne me paraît pas inutile d'insister sur le fait qu'elle est le résultat d'une utilisation attentive des possibilités propres aux instruments choisis (parce que souvent on lit dans les journaux que "ça sonne comme de la musique électronique", que l'on "voit l'influence de l'électronique", etc.). II est donc absolument faux de parler d'une transposition à l'orchestre d'effets sonores électroniques ainsi qu'on l'a fait fréquemment au cours des dernières années. Les GROUPES sont écrits pour un certain effectif orchestral et leur sonorité résulte du respect de l'égalité propre à l'utilisation fonctionnelle de cet ensemble [mot incompréhensible]. II s'agit là d'un exemple d'orchestre moderne, et cela n'a rien à voir avec la musique électronique. La composition sonore de celle-ci répond à une nécessité toute différente qui résulte de ses propres conditions d'existence. La musique électronique ne devrait point lorgner vers la musique d'orchestre et une musique d'orchestre conçue conformément aux vrais pouvoirs instrumentaux se passe aisément d'oripeaux 
pseudo-électroniques. II s'agit toujours de trouver le son "vrai", le son fonctionnel, et non pas l'effet sonore, les musiciens sont les mieux placés pour le savoir. La composition des GROUPES prévoit une disposition de l'orchestre en fer à cheval.

MK: Comme nous l'avons vu à Donaueschingen.

KS: C'est ça. Du point de vue de l'auditeur, l'un des orchestres occupe tout le côté gauche, le deuxième toute la partie devant, et enfin le troisième orchestre se trouve à droite de l'auditoire. Celui-ci se trouve ainsi complètement englobé par la disposition des trois orchestres. On peut se poser une dernière question: pourquoi accepter de diffuser par voie radiophonique des œuvres spécialement conçues pour la distribution spatiale? La réponse est toute simple: n'est-il pas préférable de voir des reproductions d'œuvres plastiques, plutôt que de n'en rien voir du tout? Peut-être ce premier contact incitera-t-il à aller voir les originaux aux endroits où ils sont exposés. Il en est de même pour des émissions radiophoniques de cette espèce.

MK: Et si vous permettez, j'ajouterai un dernier argument en faveur de la diffusion radiophonique, c'est que, d'une part, pour nous Canadiens, il n'est pas à envisager de sitôt d'avoir une exécution des GROUPES POUR TROIS ORCHESTRES, et, aussi, il y a peut-être en faveur de l'auditeur de radio un contact plus direct avec la musique

\section{KS: Oui, oui}

MK: parce qu'il est privé de l'élément visuel qui nous distrayait à Donaueschingen en quelque sorte. Ici, l'auditeur va recevoir directement votre musique Karlheinz Stockhausen, votre musique qui est interprétée par l'orchestre du Südwestfunk de Baden-Baden sous votre propre direction, conjointe à celle de Pierre Boulez et celle de Hans Rosbaud.

\section{Extrait sonore : GRUPPEN \\ für drei Orchester}

Suggestion d'écoute

GRUPPEN für drei Orchester: WDR Orchestra;

Karlheinz Stockhausen, Bruno Maderna et Michael Gielen, chefs; enregistrement de 1965;

Stockhausen-Verlag $n^{\circ} 5$.

MK: Karlheinz Stockhausen, il est rare qu'un créateur se prête aussi volontiers aux questions indiscrètes, surtout en ce qui concerne l'essence profonde de son œuvre. Vous avez bien voulu répondre à cette sollicitation de ma part parce que je vous ai expliqué que c'était essentiel pour les auditeurs du réseau français de Radio-Canada; je crois qu'ils vous seront reconnaissants avec moi de l'avoir fait et, voudriez-vous me dire si vous reviendriez parmi nous?

KS: Vous savez, je n'ai pas vu grand-chose de ce pays-là, mais je l'aime déjà. Peut-être je vais revenir, sûrement: Dans quatre ans.

MK: Alors au revoir.

KS: Bonsoir!

\section{B I B LIOG RAPHIE}

BaIL, Louise (2002), Maryvonne Kendergi - La musique en partage, Montréal, Éditions Hurtubise HMH, Cahiers du Québec, coll. «Musique», 378 p. (inclus un disque compact).

Nos plus vifs remerciements à Kathinka Pasveer (Stockhausen Foundation for Music, Kürten, Allemagne) à Maryvonne Kendergi, et au Service de la gestion de documents et des archives de l'Université de Montréal, qui nous ont permis de réaliser et publier cette transcription. Nos remerciements s'étendent aussi à la musicologue Marie-Thérèse Lefebvre, qui nous a donné accès aux informations qu'elle a recueillies sur les visites de Stockhausen à Montréal. 